\title{
A Two-sided Loop X-Ray Solar Coronal Jet Driven by a Minifilament Eruption
}

\author{
Alphonse C. Sterling ${ }^{1}$ (D) , Louise K. Harra ${ }^{2}$ (i), Ronald L. Moore ${ }^{1,3}$, and David A. Falconer ${ }^{1,3}$ \\ ${ }^{1}$ Marshall Space Flight Center, Huntsville, AL 35812, USA; alphonse.sterling@nasa.gov \\ ${ }^{2}$ UCL-Mullard Space Science Laboratory Holmbury St Mary, Dorking, Surrey, RH5 6NT, UK; 1.harra@ucl.ac.uk \\ ${ }^{3}$ Center for Space Plasma and Aeronomic Research, University of Alabama in Huntsville, Huntsville, AL 35899, USA; ron.moore@nasa.gov, \\ David.a.Falconer@nasa.gov \\ Received 2018 August 31; revised 2018 November 6; accepted 2018 November 7; published 2019 February 1
}

\begin{abstract}
Most of the commonly discussed solar coronal jets are the type that consist of a single spire extending approximately vertically from near the solar surface into the corona. Recent research supports that eruption of a miniature filament (minifilament) drives many such single-spire jets and concurrently generates a miniflare at the eruption site. A different type of coronal jet, identified in X-ray images during the Yohkoh era, are two-sided loop jets, which extend from a central excitation location in opposite directions, along low-lying coronal loops that are more-or-less horizontal to the surface. We observe such a two-sided loop jet from the edge of active region (AR) 12473, using data from Hinode X-Ray Telescope (XRT) and Extreme Ultraviolet Imaging Spectrometer (EIS), and from Solar Dynamics Observatory's (SDO) Atmospheric Imaging Assembly (AIA) and Helioseismic and Magnetic Imager (HMI). Similar to single-spire jets, this two-sided loop jet results from eruption of a minifilament, which accelerates to over $140 \mathrm{~km} \mathrm{~s}^{-1}$ before abruptly stopping after striking an overlying nearly horizontal-loop field at $\sim 30,000 \mathrm{~km}$ in altitude and producing the two-sided loop jet. An analysis of EIS raster scans shows that a hot brightening, consistent with a small flare, develops in the aftermath of the eruption, and that Doppler motions $\left(\sim 40 \mathrm{~km} \mathrm{~s}^{-1}\right)$ occur near the jet formation region. As with many single-spire jets, the magnetic trigger here is apparently flux cancelation, which occurs at a rate of $\sim 4 \times 10^{18} \mathrm{Mx} \mathrm{hr}^{-1}$, broadly similar to the rates observed in some single-spire quiet-Sun and AR jets. An apparent increase in the (line-of-sight) flux occurs within minutes of the onset of the minifilament eruption, consistent with the apparent increase being due to a rapid reconfiguration of low-lying fields during and soon after the minifilament-eruption onset.
\end{abstract}

Key words: Sun: corona - Sun: filaments, prominences - Sun: UV radiation - Sun: X-rays, gamma rays

Supporting material: animations

\section{Introduction}

Coronal jets are transient collimated ejections of solar material, typically of a length of $\lesssim 10^{5} \mathrm{~km}$ and a width of $\sim 10^{4} \mathrm{~km}$. They were first observed in detail in X-rays with the Yohkoh Soft X-ray Telescope (SXT; Shibata et al. 1992; Shimojo et al. 1996). Later they were studied with the X-Ray Telescope (XRT) on Hinode (Cirtain et al. 2007; Savcheva et al. 2007). More recently they have been studied in the extreme ultraviolet (EUV) with the Solar Terrestrial Relations Observatory (STEREO) spacecraft (e.g., Nisticò et al. 2009) and with the Atmospheric Imaging Assembly (AIA) on the Solar Dynamics Observatory (SDO; e.g., Moore et al. 2013; Sterling et al. 2015). There are many additional jet studies with a variety of instruments (Raouafi et al. 2016).

Typical jets consist of a bright base near the solar surface, with a single spire extending progressively outward from near the solar surface into the corona. (Shibata et al. 1994 called these "anemone jets"; here we will refer to them as "single-spire jets.") It was first suggested that such jets result according to an "emerging-flux model," whereby a magnetic bipole emerges from below the solar surface into the corona and undergoes magnetic reconnection with surrounding open (or far-reaching) nearly vertical field, with the jet spire forming along that open field (e.g., Shibata et al. 1992; Yokoyama \& Shibata 1995). Later, however, improved resolution and wavelength coverage with AIA showed that many (if not most) jets instead resulted from eruption of a miniature filament, or "minifilament," of size scale $\sim 10,000 \mathrm{~km}$ (Sterling et al. 2015; also see, e.g., Shen et al. 2012a; Adams et al. 2014; Panesar et al. 2016), accompanied by a "miniflare" brightening at the edge of the jet base (this brightening is sometimes called a jet(-base) bright point, or JBP; e.g., Sterling et al. 2015). Meanwhile improved magnetic field coverage with $S D O$ 's Helioseismic and Magnetic Imager (HMI) showed that often jets coincide with magnetic flux cancelation (e.g., Huang et al. 2012; Adams et al. 2014; Young \& Muglach 2014); in some cases where the flux emergence coincides with the jets, the jets still originate from locations where one pole of the emerging bipole cancels with surrounding fields (e.g., Shen et al. 2012a; Li et al. 2015). From the "minifilament-eruption model" standpoint, the flux cancelation builds the minifilament-holding magnetic field (Panesar et al. 2017) and triggers it to erupt (Panesar et al. 2016, 2018) to form the jet.

In addition to single-spire jets, there are also two-sided loop coronal jets (Shibata et al. 1994), whereby two spires develop roughly symmetrically and horizontally to the surface from a central bright region. These two-sided loop jets were first seen in coronal X-ray images and are also seen in EUV images (e.g., Alexander \& Fletcher 1999; Jiang et al. 2013). The emergingflux model was also invoked to explain these jets theoretically, with the emerging-flux reconnecting with overlying horizontally directed fields; numerical simulations of this showed results similar to the Yohkoh/SXT X-ray observations (Yokoyama \& Shibata 1995, 1996). Published examples of SDO-era observations, with high-resolution and high-cadence multi-wavelength images and magnetograms, of these two-sided loop jets are sparse (see, however, Section 4 for recent references). Here we present Hinode and $S D O$ observations that show strong 
evidence that a two-sided loop jet resulted from an erupting minifilament, similar to that in single-spire jets.

\section{Instrumentation and Data}

Our observed two-sided loop jet occurred on 2015 December 30 near 22:41 UT, southeast of a set of sunspots in National Oceanic and Atmospheric Administration (NOAA) active region (AR) 12473 that was located at heliocentric latitude and longitude of about $-20,+45$, producing a C-level enhancement in the Geostationary Operational Environmental Satellite (GOES) X-ray flux.

We use imaging data from both XRT and AIA. We confirm that it is an "X-ray jet" with XRT, assuring that we are studying a two-sided loop jet similar to those identified by Shibata et al. (1994) in X-rays with SXT. XRT has spatial pixel resolution of 1 !"02, with a variable time cadence and field of view (FOV; Golub et al. 2007). AIA produces full-Sun images in seven EUV bands at $12 \mathrm{~s}$ cadence with 0!" 6 pixels (Lemen et al. 2012); for this study we examined all EUV channels and the $1600 \AA$ UV channel. For our purposes here, we find it adequate to present 304, 193, 211, and $94 \AA$ images (and their $12 \mathrm{~s}$ cadence animations), which have peak contributions at $5 \times 10^{4}, 1.6 \times 10^{6}, 2.0 \times 10^{6} \mathrm{~K}$, and $6.3 \times 10^{6} \mathrm{~K}$, respectively. We also use raster-image data from the Hinode/EUV Imaging Spectrometer (EIS, Culhane et al. 2007) in the He II $304 \AA$, Fe XII $195 \AA$, and Fe XV $284 \AA$ lines. Each scan required $\sim 3.5 \mathrm{~min}$ to cover an east-west extent of $\sim 50^{\prime \prime}$, with a north-south slit extent of $\sim 120^{\prime \prime}$. There was a fortuitous overlap with the jet event in the southwest quadrant of EIS's limited FOV. We also use magnetograms from HMI (Scherrer et al. 2012), which has a maximum cadence of $45 \mathrm{~s}$ and 0 ." 5 pixels.

\section{Observational Results}

\subsection{X-Ray and EUV Evolution}

Figures 1(a)-(b) show the two-sided loop jet in XRT images with classic morphology (Shibata et al. 1994; Yokoyama \& Shibata 1995, 1996) in X-rays, with a strong brightening inbetween the two loop-confined arms (spires) of the jet; the accompanying animation shows the jet's evolution. These Hinode/XRT images are very close in appearance to the snapshot from Yohkoh/SXT in Yokoyama \& Shibata (1995; see Figure 3(b) of that paper; that figure is also in Shibata et al. 1994). Both the Yokoyama \& Shibata (1995) jet and our jet have one side that is smaller and brighter (to the south in the Yokoyama \& Shibata 1995 jet, and to the northeast in ours), and one side that is larger and dimmer (north in Yokoyama \& Shibata 1995, and southeast in ours). Viewing the animation accompanying our Figures 1(a)-(b), the frame at 22:46:10 UT comes closest in appearance to the snapshot in the Yokoyama \& Shibata (1995) figure (we choose to show different frames in our Figures 1(a) and (b) because of the strong saturation of the flaring location in that 22:46:10 UT frame). As with the original observations with Yohkoh, however, the X-ray images alone yield little direct information about the cause of the jet. We look to additional data for more insight.

Figures 1(d)-(f) show the jet with AIA $94 \AA$ images, with an initial brightening in Figure 1(d) (also visible in X-rays in Figure 1(a)), the two-sided loop jet structure in Figure 1(e), and a close-up in Figure 1(f). Arrows in Figure 1(e) point to oppositely directed flows along two strands of the two jet-guiding lobes.
Figures $1(\mathrm{~g})-(\mathrm{h})$ show the event in AIA $193 \AA$, with arrows in Figure 1(g) showing the elevated field that later becomes one of the jet-guiding lobes. Figure 1(i) shows a close-up of the region that brightens at the earliest sign of jet activity, with a filament-like absorbing feature (arrows) that the accompanying animation shows to be erupting. The erupting segment (black arrow) has length of $\gtrsim 15^{\prime \prime}(\approx 11,000 \mathrm{~km})$. This is similar to the size $(8000 \mathrm{~km})$ Sterling et al. (2015) found for erupting minifilaments when they were at about the same relative distance above the surface as the minifilament in Figure 1(i). This is smaller than the sizes of typical filaments ( 30,000-110,000 km; Parenti 2014). Thus, just as with the more typical "single-spire" jets, this twosided loop jet apparently results from an erupting minifilament. From the accompanying animations, the jet forms when this erupting minifilament collides with pre-existing overlying field, as shown by arrows in Figure 1(g).

Figure 2 shows AIA $94 \AA$ images with a larger FOV than Figure 1, thereby more clearly revealing the overall jet structure. After an initial brightening (the red arrow in Figures 2(a) and (c)), the jetting into the two lobes eventually extends out asymmetrically from that brightening, longer toward the southeast and shorter toward the northwest (Figure 2(c)). We see the southeast lobe develop in time in a similar fashion in several AIA wavelengths; in these $94 \AA$ images the jet-spire brightening in the lobe, indicated by the white arrow in Figure 2(b), extends away from the initiation site at $\sim 550 \pm 100 \mathrm{~km} \mathrm{~s}^{-1}$. In contrast, the northeast lobe appears to brighten mostly in place, and therefore seems to be mostly a result of material entering into the passband of sensitivity for the $94 \AA$ wavelength channel as it heats or cools, rather than arising from a front propagating along the field to fill the loop. Because of the differences in temperature response in the $94 \AA$ EUV of AIA (Lemen et al. 2012) and the broadband X-rays of XRT (Golub et al. 2007), jets can appear differently in the different wavelength regimes (see Sterling et al. 2015). This is the case here; for example the northwestern lobe of the jet is clearly visible from the earliest XRT images (22:44:09 UT), while it does not appear until substantially later in $94 \AA$ (the orange arrow in Figure 2(c)). Evolution of the southwest lobe is similar in X-rays to that in $94 \AA$, with flows along the lobe field from the central region visible over 22:44-22:48 UT, approximately coinciding with the flows visible in the $94 \AA$ images.

Two-sided loop jets (and single-spire jets too, for that matter) were originally defined from X-ray images, and thus the images expected to match most closely those in the sketches of Shibata et al. (1994) and Yokoyama \& Shibata $(1995,1996)$ are those of Figures 1(a) and (b), although unfortunately this appearance is compromised somewhat by the limited FOV of our XRT images in Figure 1.

Figure 3 shows a zoomed-in view of the erupting minifilament, this time in AIA $304 \AA$ images. Over some time the minifilament becomes visible near the surface (Figure 3(a)). Figure 3(b) shows a brightening beneath the minifilament as it has just started to rise; this brightening is visible in all AIA channels, and so includes hot emissions. A short time later (Figure 3(c)) the brightenings have become much more pronounced. Moreover, the minifilament itself now shows a contorted, serpentine-like shape-likely due to writhing - as it erupts away from the surface (Figure 3(d)). At the time of Figure 3(e) the erupting and expanding minifilament reaches the pre-existing overlying field shown by the white arrows in Figure 1(g) and by light-blue arrows in Figures 3(d)-(f). From the corresponding animation, upon pushing into the overlying 


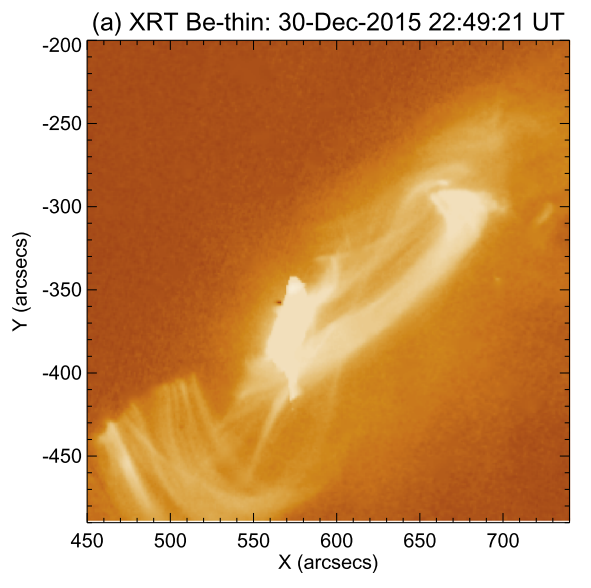

(d) AIA 94: 30-Dec-2015 22:43:48 UT

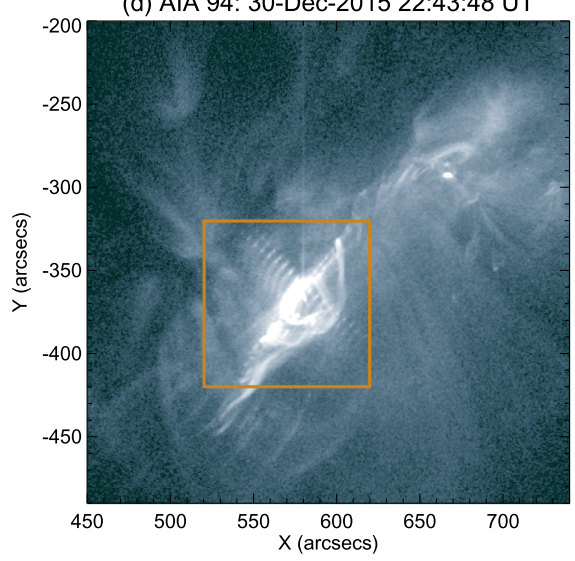

(g) AIA 193: 30-Dec-2015 22:42:41 UT

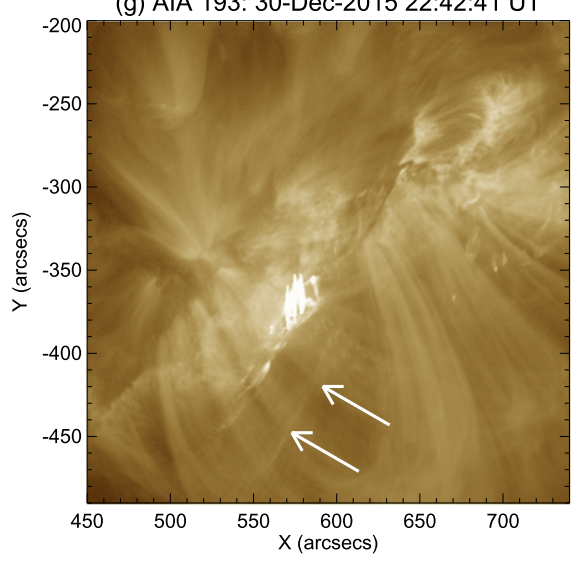

(b) XRT Be-thin: 30-Dec-2015 23:17:22 UT

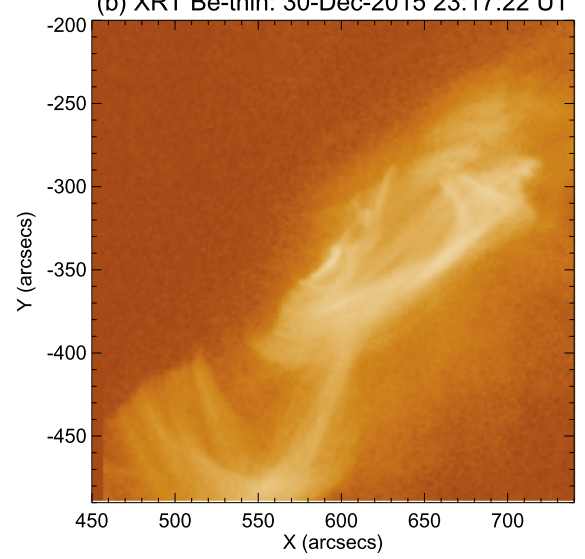

(e) AIA 94: 30-Dec-2015 22:49:00 UT

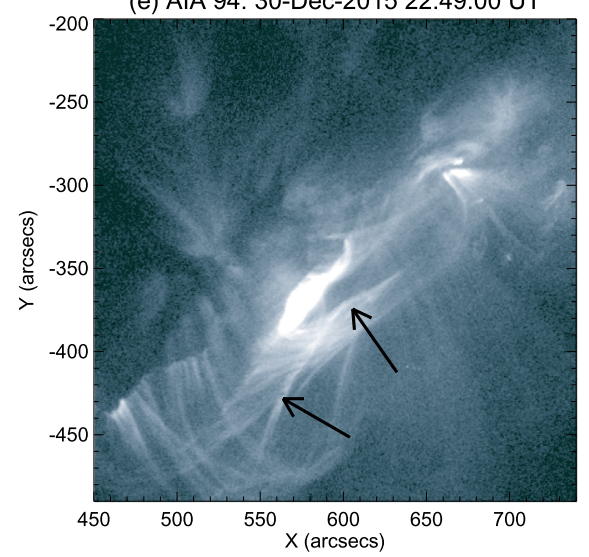

(h) AIA 193+HMI: 30-Dec-2015 22:43:43 UT

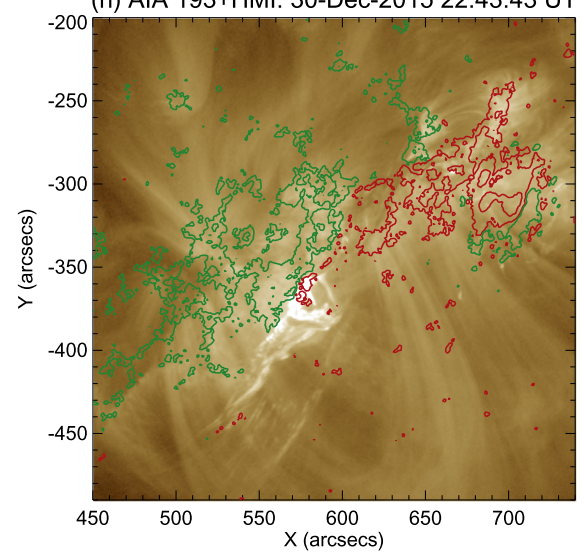

(c) HMI: 30-Dec-2015 23:43:08 UT

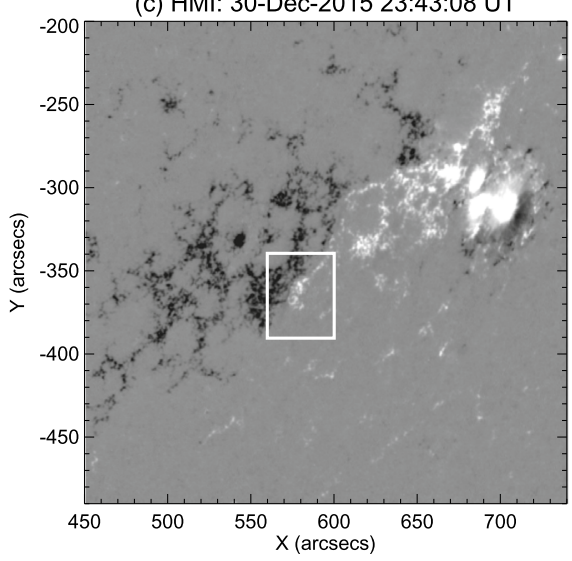

(f) AIA 94: 30-Dec-2015 22:43:48 UT

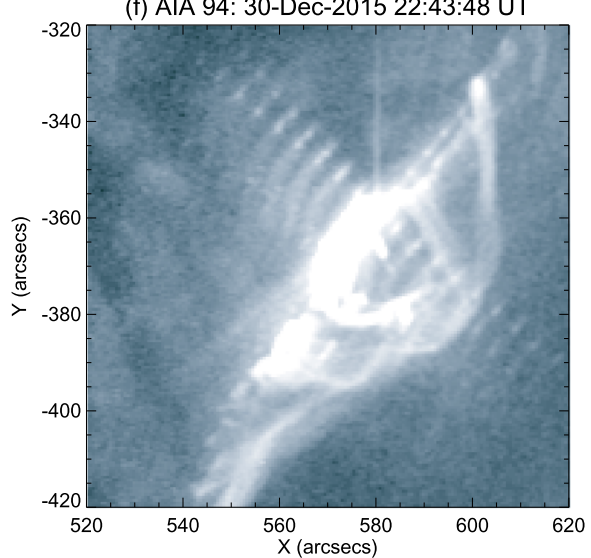

(i) AIA 211: 30-Dec-2015 22:43:25 UT

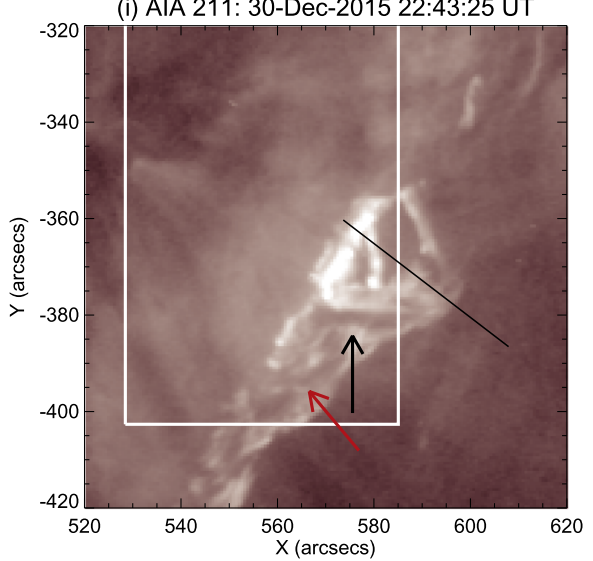

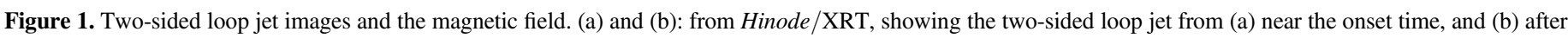

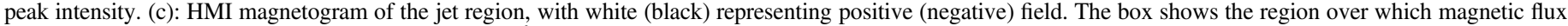

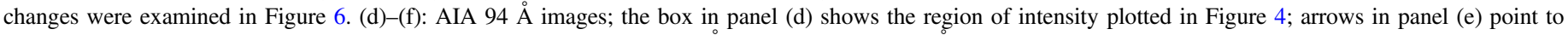

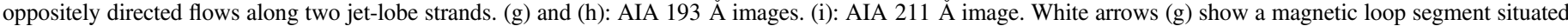

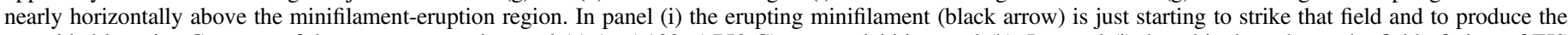

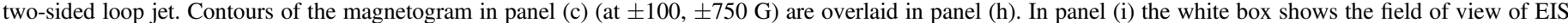

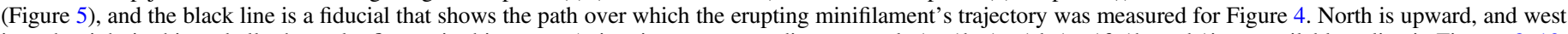

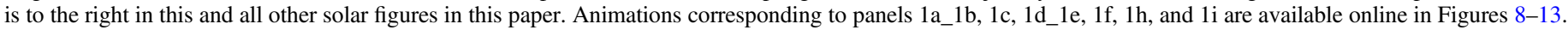

field, the minifilament appears to show untwisting motions over approximately 22:44-22:48 UT. Along with and continuing after this unwinding, flows toward the northwest occur from the erupting minifilament (the green arrow in Figures 3(e)-(f)). Also, the overlying feature (light-blue arrows) shows distinct flows southeastward from approximately the time it is impacted by the erupting-minifilament field, at about 22:45 UT, and continues until about 22:51 UT in the animation. These features are also visible in the other AIA EUV channels; see the animations accompanying Figure 1.

Figure 4 shows the trajectory of the erupting minifilament as a function of time, where we have visually estimated the top of 

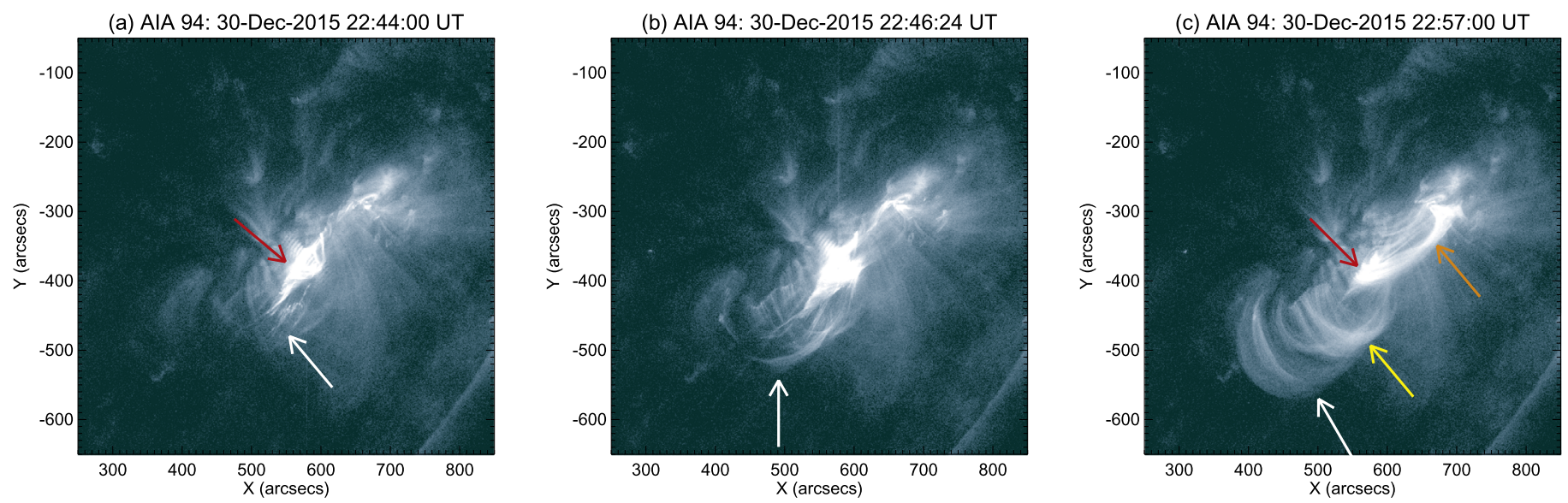

Figure 2. AIA $94 \AA$ Amages showing a larger FOV perspective of the two-sided loop jet of Figure 1. (a) After the initial brightening from the jet initiation region (red arrow), a lobe of the jet starts to appear (white arrow). (b) The lobe to the east continues to evolve toward the southeast (white arrow). (c) The full structure of the twosided loop jet. One lobe extends to the southeast, in this case along multiple field lines, with the white and yellow arrows pointing to two such groups of field lines. A second lobe extends to the northwest (orange arrow); this lobe was prominent from an earlier time in the jet's development in the X-ray images (Figures 1(a) and (b)), but is just now becoming apparent in $94 \AA$. Jets such as these are called two-sided loop jets due to their roughly symmetric structure around the bright point at the initiation location (red arrow). Animation 1d_1e (Figure 10) shows this sequence.

(a) AIA 304: 30-Dec-2015 22:33:30 UT

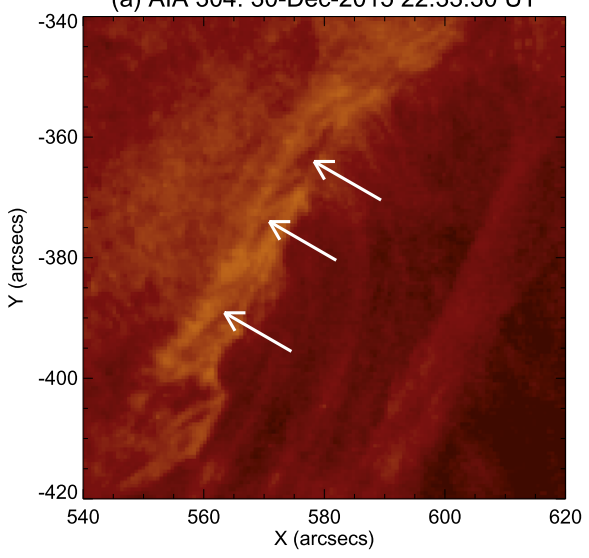

(d) AIA 304: 30-Dec-2015 22:43:30 UT

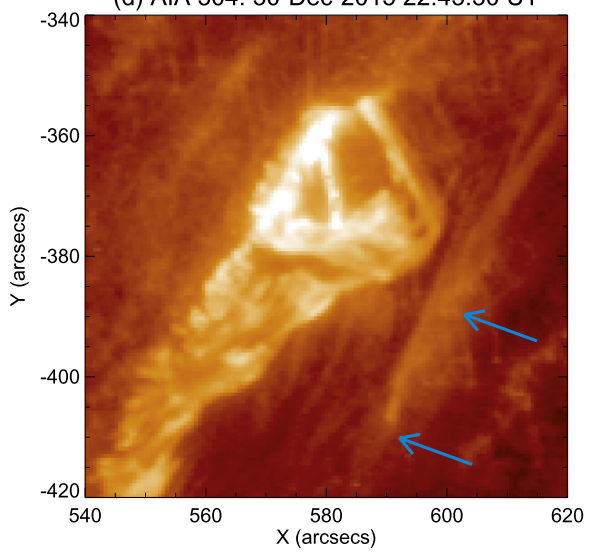

(b) AIA 304: 30-Dec-2015 22:36:54 UT

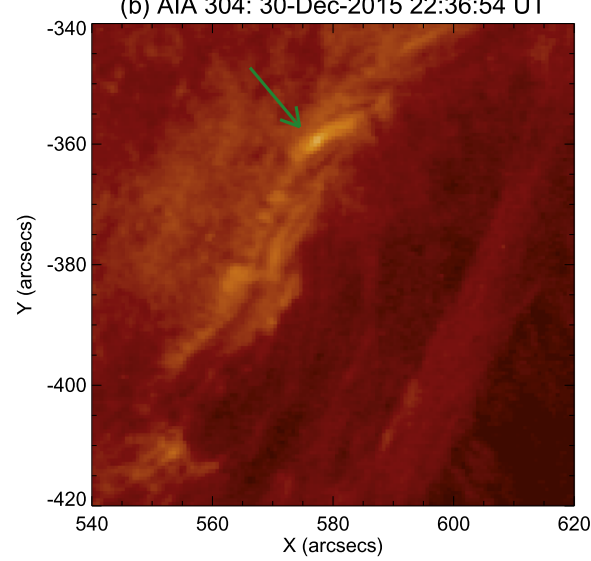

(e) AIA 304: 30-Dec-2015 22:45:06 UT

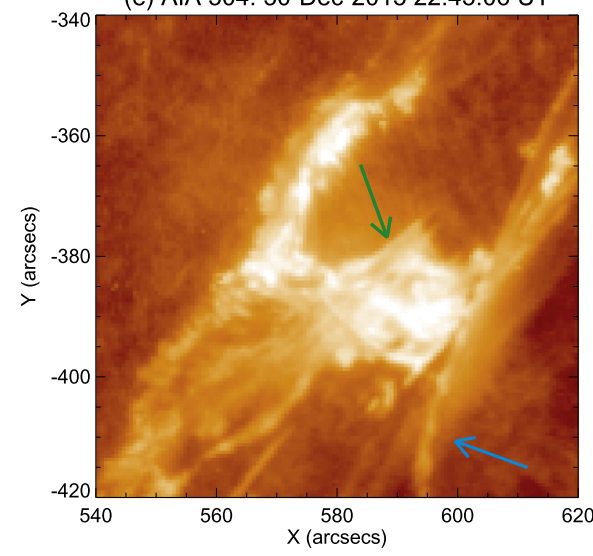

(c) AIA 304: 30-Dec-2015 22:42:42 UT

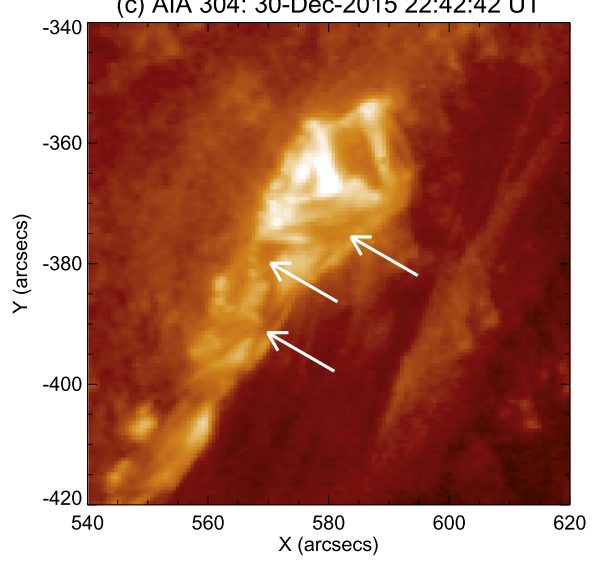

(f) AIA 304: 30-Dec-2015 22:46:42 UT

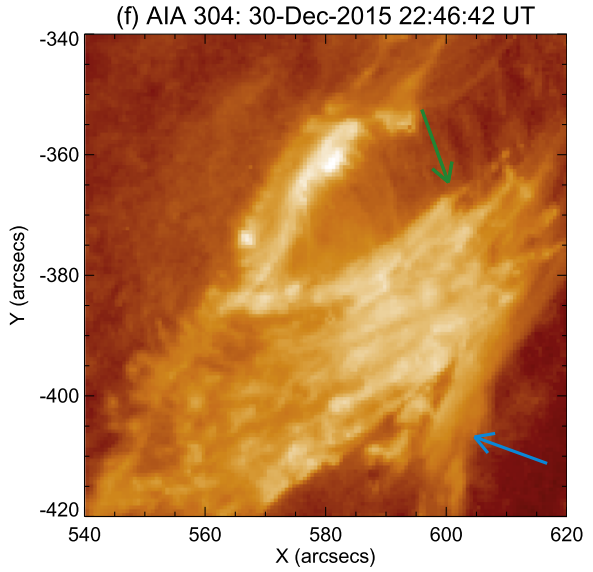

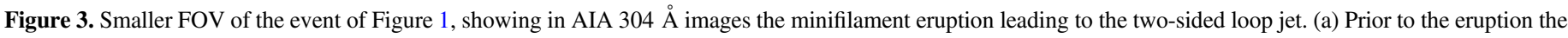

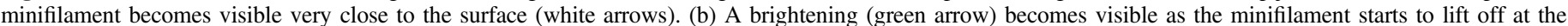

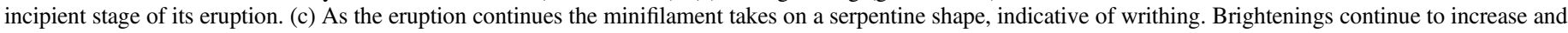

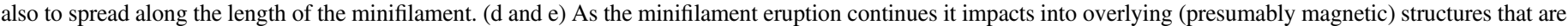

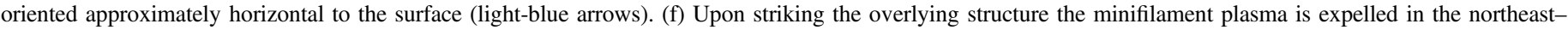

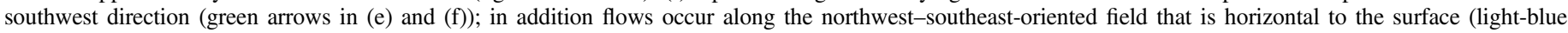
arrows). These motions are apparent the online animated Figure 14. 


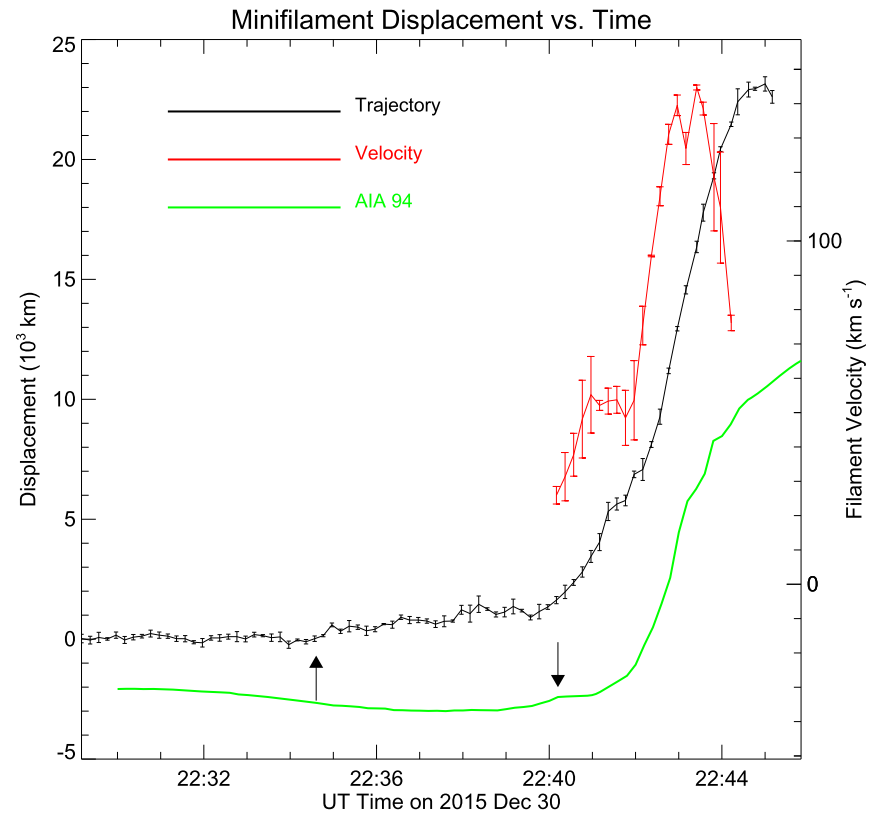

Figure 4. Characteristics as functions of time of the minifilament that erupts and drives the two-sided loop jet. (Black line) Height of top of minifilament segment along the path of the black fiducial line in Figure 1(i). Error bars are $1 \sigma$ uncertainties from three independent measurements. (Orange line) Velocities derived from the height trajectory (smoothed over four time steps $=48 \mathrm{~s}$ ). (Green line) Intensity from the AIA $94 \AA$ channel, integrated over the region of the orange box in Figure 1(d). The arrow on the left shows the start of a pre-eruption rise in the minifilament's height. The arrow on the right shows a minor peak in the $94 \AA$ intensity, which coincides with the onset of a faster rise; the $94 \AA$ animation accompanying Figure 1 indicates a corresponding intensity brightening at the time for the left arrow as well, but that peak in not visible in the green curve due to enhanced background emission.

the minifilament as it moves upward in $211 \AA$ images (Figure 1(i)). We perform each length measurement three independent times to estimate the random uncertainty in the measured value. Upward movement of the central part of the filament commences near 22:34 UT, indicated by the upwardpointing arrow. This is the time of the initial brightening at the base of the minifilament indicated by the green arrow in Figure 3(b); it is also visible in the AIA animations accompanying Figure 1, for example in the $94 \AA$ animation of Figure 1(f) from 22:34:24, and especially at 22:35:24 UT. In Figure 4 the green curve represents the AIA $94 \AA$ flux integrated over the region of the box in Figure 1(d), but the 22:34:24 UT flux increase visible in the animation is too weak, relative to the background intensity, to stand out in the green light curve. (The enhanced background intensity in the $94 \AA$ images, visible as a hump in the green curve of Figure 4 that peaks near 22:30 UT, is caused by other dynamical processes that we do not investigate here.) From about 22:40 UT there is a sharp increase in the rise trajectory of the minifilament, and this corresponds to an increase in the $94 \AA$ integrated intensity (the downward-pointing arrow in Figure 4, visible over 22:39-22:41 UT in the animation accompanying Figure 1(f)), and then a further acceleration of the minifilament near 22:42 UT, indicated by the velocity plot (orange) in Figure 4, which is also accompanied by a sharp increase in the integrated $94 \AA$ intensity. This latter-most sharp increase in the $94 \AA$ intensity can be considered to be the growth of a small flare arcade accompanying the accelerated rise of the minifilament.
This behavior of the minifilament's rise, with a slow-rise phase prior to the most-accelerated rise, EUV brightening enhancements accompanying accelerations in the rise trajectory, and the onset of strongest flaring accompanying the strongest minifilament upward acceleration all closely mimic behavior of larger-scale erupting filaments and their accompanying flares (e.g., Sterling \& Moore 2005; Sterling et al. 2007, 2014; Imada et al. 2014; McCauley et al. 2015; Green et al. 2016; Harra et al. 2017).

From Figure 4 the minifilament erupts outward until it reaches a height (displacement) of about 23,000 km, when it abruptly stops, apparently arrested by overlying field that is visible even prior to the jet (arrows in Figure $1(\mathrm{~g})$ ). Due to the projection angle, the true height might be $\sim 30 \%$ larger than these plane-of-sky projected values, as the event occurred at an angle of $\sim 45^{\circ}$ with the Earth-Sun line of sight; so the height is $\sim 30,000 \mathrm{~km}$. A comparison with previous studies of ejective eruptions (e.g., Sterling et al. 2014) suggests that the minifilament's rise is relatively unimpeded until the abrupt velocity decrease near 22:44 UT, when animations accompanying Figure 1 indicate that it pushes into the overlying magnetic field.

\subsection{EIS Rasters}

Figure 5 shows an EIS raster intensity image in He II (Figure 5(a)) and a ratio of Fe XV-to-Fe XII intensity images (Figure 5(b)). These ratio images provide a qualitative measure of hotter (brighter) and cooler (darker) locations in the region (Doschek et al. 2007). Figure 5(c) shows contours from Figure 5(b) on an AIA 193 image. The rectangle in Figure 1(i) shows that the EIS FOV covers only a small portion of the jet's base region.

Figure 5(a) shows a dark feature (light-blue arrow) and a bright feature (black arrow), which correspond to cooler and hotter locations in Figure 5(b). Comparing these features with the AIA features in the zoomed-in animations corresponding to Figures 1(f), (i), and Figure 3 suggests that the dark feature could be a void; see, for example, the Figure $3304 \AA$ animation between 22:42 and 22:45 UT, where there seems to be a region comparatively free of emitting material between the erupting minifilament and the photosphere at the location of the EIS dark feature. For the bright feature (black arrow in Figure 5(a)) a comparison with the same animations shows convincingly that this region corresponds to flare loops that develop below the erupting minifilament; see, for example, the $211 \AA$ animation corresponding to Figure 1(f) from about 22:50 UT, when the features at this location take on the distinct appearance of post-flare loops. Unfortunately, however, the EIS scans did not capture the erupting minifilament at a time when it was most clearly visible in the AIA images, such as in Figure 1(i). There is only one EIS scan coinciding with times where AIA shows the minifilament very clearly in the EIS FOV; that is at 22:42:18 UT. But that scan has a data dropout near the jet region, and so we are not confident in that scan. At the time of the prior raster $(22: 38: 44 \mathrm{UT})$ the minifilament has not yet started lifting off, and by the time of the next scan (22:45:52 UT), hotter material envelopes the minifilament, which is apparent in the AIA animations accompanying Figure 1. So we can say with certainty from the EIS rasters is that they show: (a) brightening beneath the erupting minifilament, (b) temperature-ratio maps showing hotter plasma at the expected small flare location, and (c) Doppler 


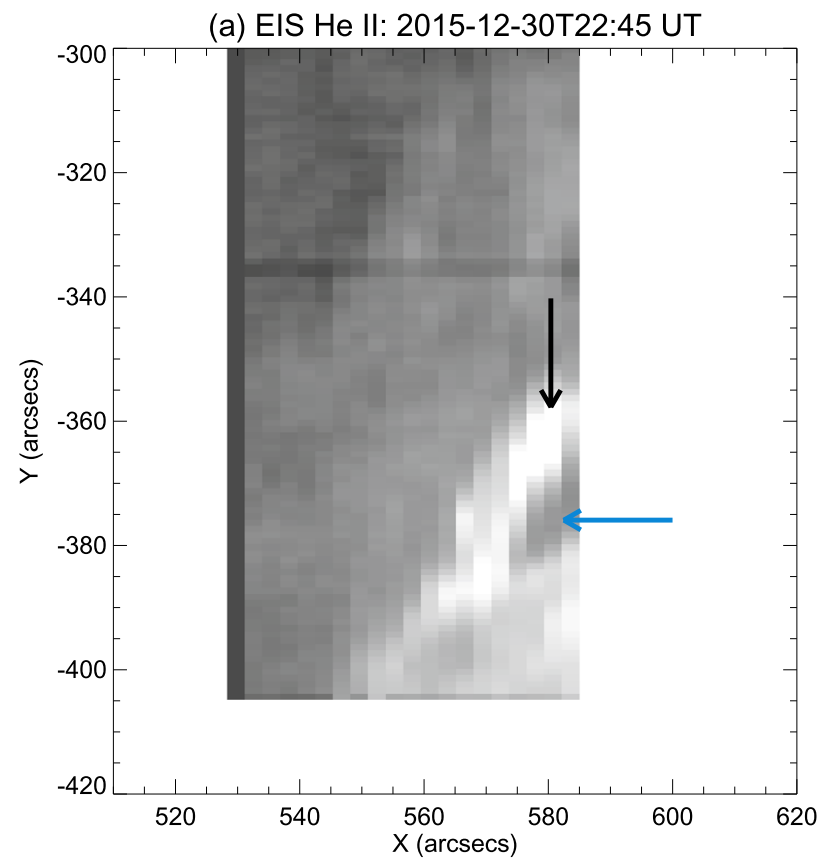

(c) Fe XV/XII on AIA 193: 30-Dec-2015 22:45 UT

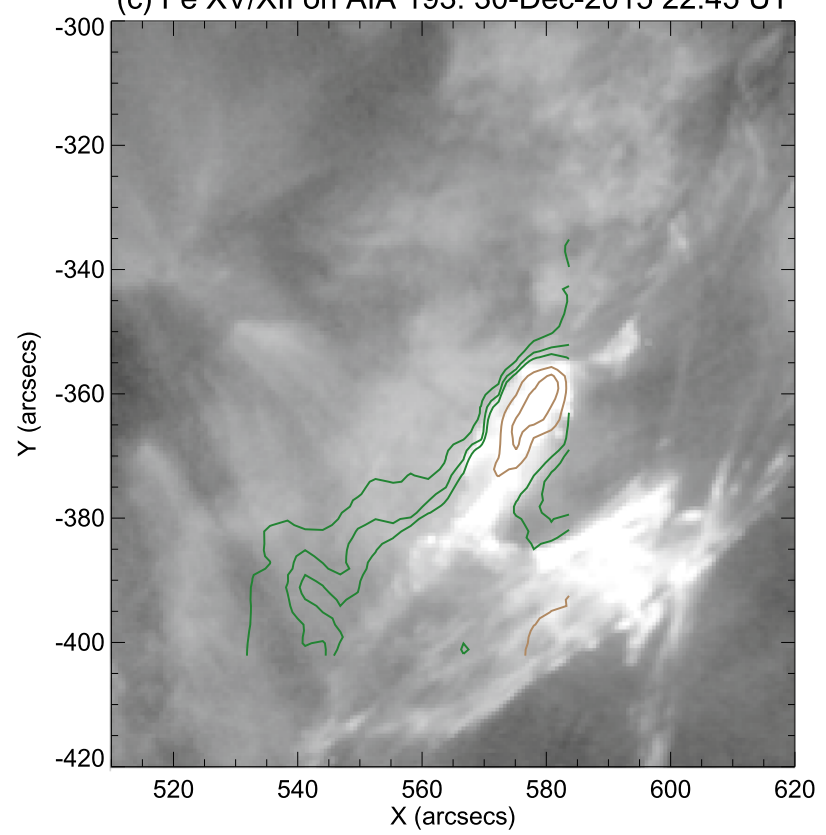

(b) EIS Fe XV/XII Ratio: 2015-12-30T22:45 UT

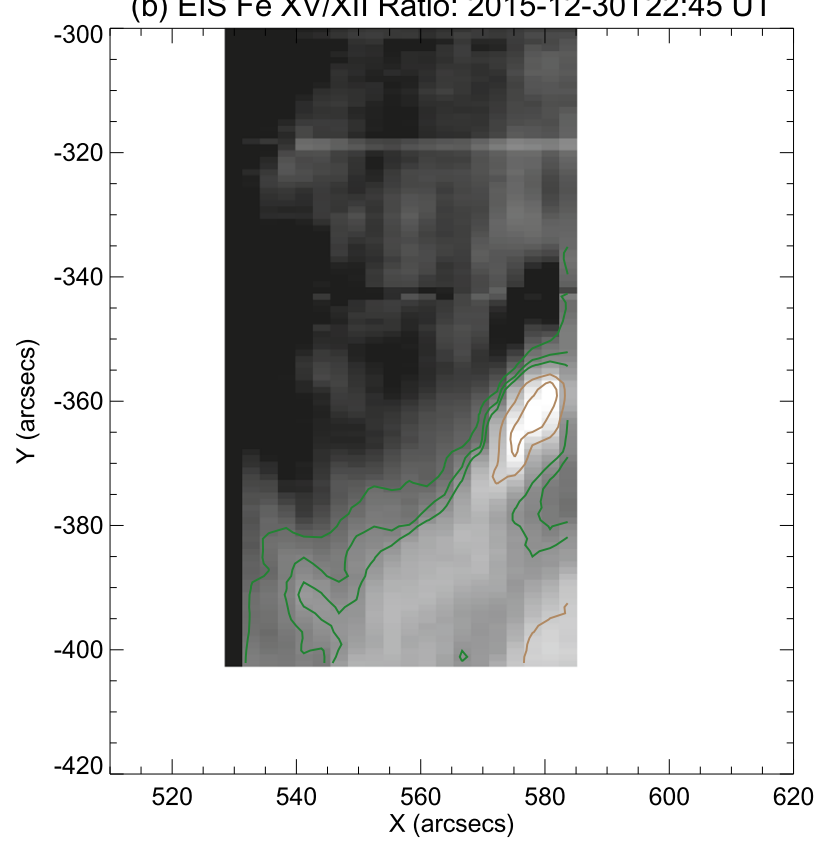

(d) Fe XII vel on AIA 193: 30-Dec-2015 22:45 UT

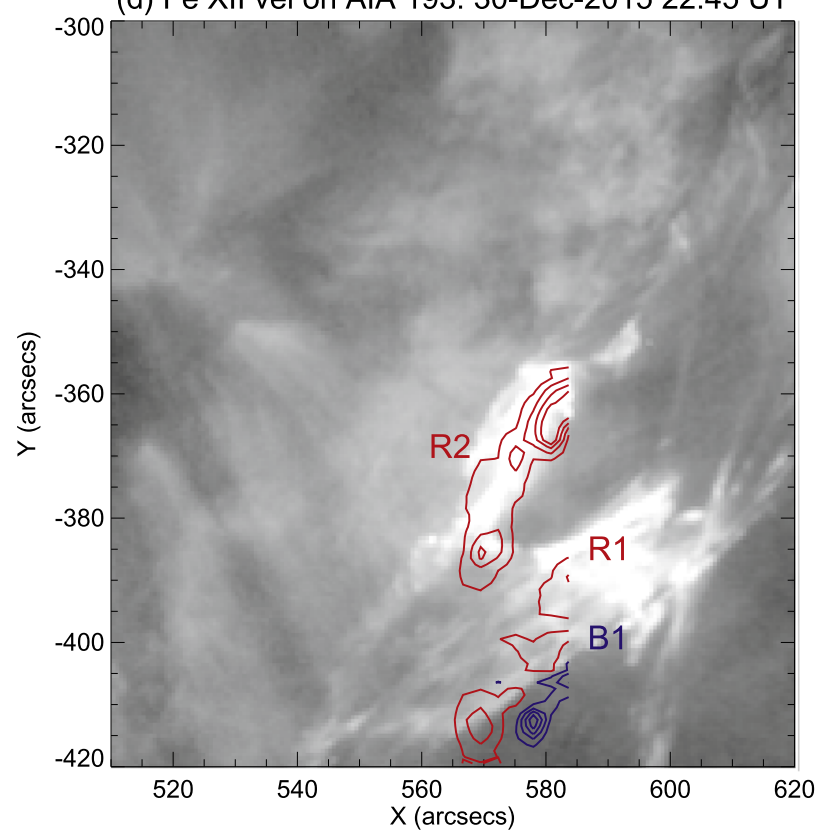

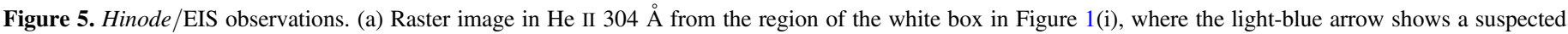

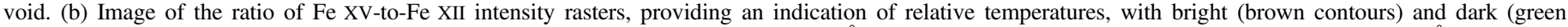

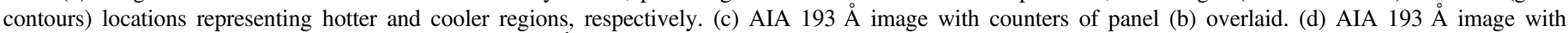

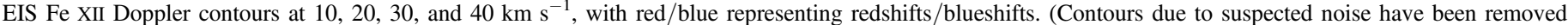
from $b-d$.)

velocities from single-Gaussian fits in several rasters show redshifts, and one raster (beginning at 22:45:52) shows a strongly blueshifted feature too (both red- and blueshifts have maximums of $\sim 40 \mathrm{~km} \mathrm{~s}^{-1}$ ); we consider these line shifts further in Section 4.

\subsection{Magnetic Evolution}

Figure 1(c) shows an HMI magnetogram of the region, and Figure 1(h) shows it overlaid onto an AIA $193 \AA$ image. From the latter panel and accompanying animation, the minifilament eruption clearly occurs on a magnetic neutral line.
Figure 6 shows the positive-polarity flux summed over the box in Figure 1(c) for the $36 \mathrm{hr}$ beginning at 0:00 UT on December 30. There is a general flux decrease over that time period of $\sim 4 \times 10^{18} \mathrm{Mx} \mathrm{hr}^{-1}$, which is in between flux-cancelation rates found in (single-spire) quiet-Sun and AR jets (see Table 1 of Sterling et al. 2018). There is, however, a prominent jump of about $4 \times 10^{19}$ Mx over $\sim 15$ minutes, beginning nearly exactly with the onset of the minifilament's eruption. This does not have the appearance of flux emergence; if it were emergence, the emergence rate during the jump would be at or above $\sim 1 \times 10^{20} \mathrm{Mx} \mathrm{hr}^{-1}$, which is the approximate maximum rate of $\mathrm{AR}$ growth 


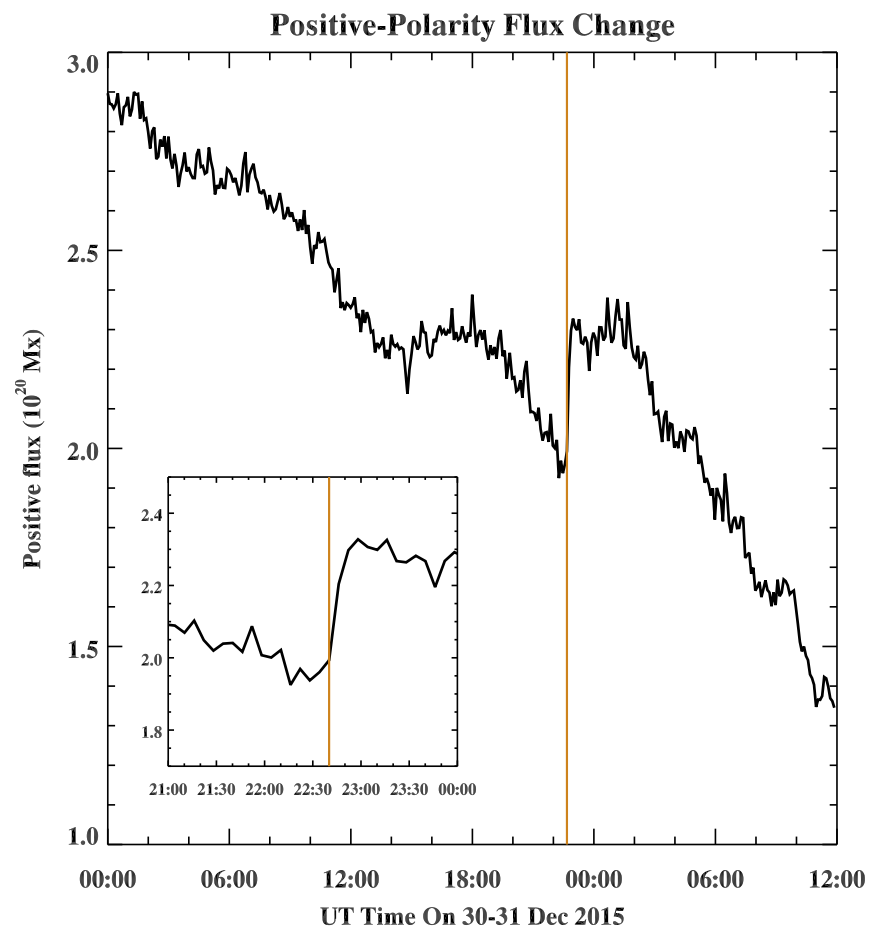

Figure 6. Positive magnetic flux, measured over the box of Figure 1(c). The orange line is at 22:40:08 UT, which is near the minifilament-eruption-onset time. The insert plot is a close-up showing that the jump in the flux value occurs very close to the time of the jet.

(Zwaan 1987); the magnetograms show no indication of such a large emergence. Instead we suspect that we are seeing an increase in the line-of-sight component of the field, as the field vector at the surface changes its direction as the minifilament erupts; the positive-polarity roots of that field were oriented such that, in response to the eruption, the direction of the field turned toward our observation direction, which is perhaps a natural consequence of the our perspective of viewing the region near the west limb (see the discussion of Figure 7 below). Similar magnetic rapid reconfigurations have been seen in flares (e.g., Moore et al. 1984; Wang et al. 1994). The continuation of the flux decrease with time for more than $12 \mathrm{hr}$ following the jet is also supportive of this hypothesis (Figure 6). Hence the evidence is that flux cancelation triggered the minifilament's eruption that drove the two-sided loop jet, consistent with flux cancelation triggering many or most single-spire jets (e.g., Panesar et al. 2016, 2017, 2018; Sterling et al. 2016, 2017).

\section{Summary and Discussion}

We find that a two-sided loop jet results from eruption of a miniature filament. This is consistent with earlier observations that minifilament eruptions cause single-spire jets. In this case, the erupting minifilament reached a velocity of $\sim 140 \mathrm{~km} \mathrm{~s}^{-1}$ prior to impacting and reconnecting with an overlying, roughly horizontal portion of a sigmoid coronal-loop field at a height of $\sim 30,000 \mathrm{~km}$. Reconnection between the erupting minifilament (more specifically, with the erupting-minifilament flux-rope field that holds the cool minifilament material) and that horizontal-loop field resulted in heating at the reconnection location and expulsion of X-ray/EUV jets in both directions along the loop, producing the two-sided loop jet. EIS raster scans confirm accentuated heating at the suspected small flare (a)

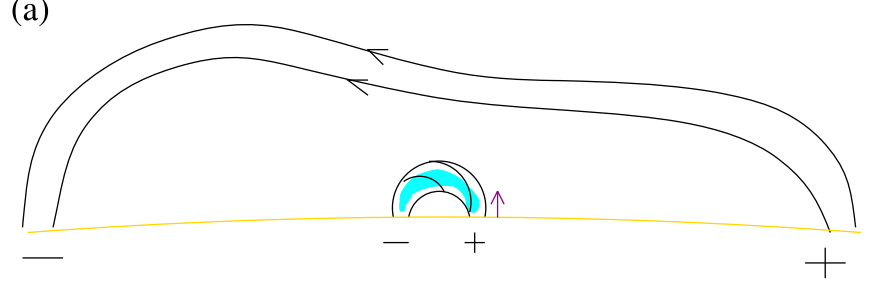

(b)

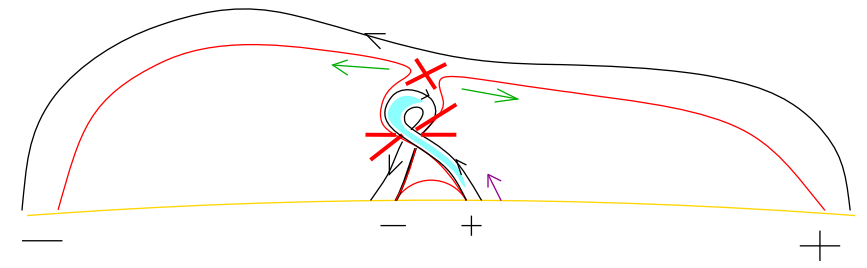

Figure 7. Schematic showing inferred production of the two-sided loop jet. (a) Setup prior to jet initiation, with the yellow curve representing the solar surface, black lines representing magnetic field lines, and the blue feature representing a cool minifilament suspended low inside of a twisted magnetic loop. Southeast is to the left, and northwest is to the right when comparing with the observations in Figures 1 and 5. (b) Upon erupting, the minifilament field writhes enough to reconnect with the overlying field. Red lines indicate the reconnected field, and red X-es represent reconnection locations. Reconnection also occurs between the legs of the writhing field, producing the red semicircular loop at the base; this is the brightening boxed in Figure 1(d) and corresponds to the JBP in the minifilament-eruption picture of Sterling et al. (2015). The green arrows represent flows in both directions along the two-sided loop jet. The purple arrows show the orientation of the positive field direction at the surface and inside of the erupting-minifilament loop, with the arrow drawn outside of that loop for clarity. If, for example, the eruption is observed from the top left corner with a line-of-sight magnetograph, this positive component of the field will appear to be larger in (b) than in (a), due to the field direction changing during the eruption; this change might explain the jump in the measured positive magnetic flux at the time of the minifilament eruption displayed in Figure 6.

location, and line-of-sight magnetogram observations are consistent with flux cancelation being the trigger for the onset of the minifilament's eruption.

Figure 7 shows a schematic of our minifilament-eruption scenario for producing this two-sided loop jet. The minifilament flux rope writhes as it erupts, allowing reconnection at the elevated location (Figure 7(b)). This schematic is topologically the same as that drawn for single-spire jets in Sterling et al. (2015), but with the "open" field now as the long horizontal loop, running from right to left in the figure (northwest to southeast in the observed jet in Figure 1).

Figure 7 also illustrates, with the purple arrows, our idea for the positive-flux jump at and soon after the time of the minifilament-eruption onset. These arrows represent the direction of the positive component of the photospheric field inside the footpoint of the minifilament loop (in the figures, for clarity we draw the arrows just outside of the minifilament loop). Prior to the eruption of the minifilament, the direction of the field vector in the minifilament's positive-polarity flux patch is approximately vertical to the surface. Within a few minutes following the minifilament-eruption's onset, however, the foot of the positive-polarity field that undergoes the flarearcade-building reconnection points more toward the top left of the schematic. In other words, after the eruption's onset, the 
purple arrow in Figure 7(b) is no longer vertical, as it was in Figure 7(a); rather it is now leaning slightly counterclockwise to the normal to the surface, pointing to the southeast (in the plane of the figure). This is a result of the reconnecting field beneath the erupting minifilament collapsing in upon itself. (We speculate that this photospheric field direction change could be a by-product of the so-called "Hudson effect," from the work of Hudson 2000; e.g., Janse \& Low 2007; Shen et al. 2012b; Panesar et al. 2013; Wheatland et al. 2018.) To an observer viewing from the top left of the schematic, the measured intensity of the line-of-sight component of the foot of that positive-polarity field would increase between the times of Figures 7(a) and (b). Because the left side of the schematic represents southeast in the solar images of this paper, and because the region appears near the solar west limb viewed from HMI, the purple arrow pointing more toward the top left in Figures 7(b) compared to 7(a) is qualitatively consistent with the observed positive-flux increase during the time of minifilament eruption. This same process would affect the negative-flux values also, but as mentioned earlier, we do not measure the negative flux here because it is difficult to isolate. While further investigation of this idea is beyond the scope of the current paper, this scenario plausibly explains how there could be the observed increase in the line-of-sight positive flux starting at the time of the jet onset shown in Figure 6 .

Overall, we observe persistent flux cancelation over the time leading up to the jet. Thus our findings are fully consistent with other recent studies showing that minifilaments that erupt to form jets are frequently built by flux cancelation and that continued cancelation triggers them to erupt; see Panesar et al. (2016, 2017, 2018) for schematic illustrations on how this buildup and triggering might occur. See, e.g., Sterling et al. $(2015,2017)$ for schematic illustrations on how the erupting minifilament would form the jet.

EIS's FOV (Figure 5(d); also see the white box in Figure 1(i)) covers the location just to the east of the interaction location of the erupting minifilament and the overlying field. In Figure 5(d) the line shifts labeled R2 are located near the AIA flare loop tops, so if they were due to downflows from the tops of those loops, we would expect a mixture of redshifts and blueshifts; but we see only redshifts here. Instead, the R2 redshifts are more consistent with material trapped in contracting reconnected closed fields, producing "supra-arcade downflows," (e.g., McKenzie 2000; Savage \& McKenzie 2011; Warren et al. 2011; Savage et al. 2012), which have minimum velocities near flare loop tops close to those observed here $\left(\sim 40 \mathrm{~km} \mathrm{~s}^{-1}\right)$. Hence we expect the source of these redshifts to be different from those seen by EIS in AR loops (e.g., Del Zanna 2008; Doschek 2012).

For the R1/B1-labeled red/blue pair of Doppler shifts in Figure 5(d), which also reach $\sim 40 \mathrm{~km} \mathrm{~s}^{-1}$, Figures 3(e)-(f) and the accompanying animation (and also other AIA animations from Figure 1 showing the minifilament-eruption region close up) show that complex dynamics ensue when the minifilament pushes into the overlying horizontal field. As mentioned in the discussion of Figure 3 in Section 3.1 the erupting minifilament shows what appears to be untwisting motions (this would correspond to the minifilament flux rope with blue shading in Figure 7(b)), along with northwestwarddirected motions (the green arrow in Figures 3(e)-(f)) and also southeastward-directed motions along the overlying horizontal field (light-blue arrows in Figures 3(d)-(f)). Of course "northwestward" and "southeastward" only describe plane-ofsky motions, but we could expect corresponding components either into or out of the plane-of-sky as well. EIS is apparently observing Doppler shifts from these opposite-directed motions; thus the shifts could be from some combination between the possible untwisting motions (as reported in, e.g., Williams et al. 2011) and the observed counterstreaming. Due to the limited FOV coverage and the limited number of scans, however, we are not able to specify more exactly what the cause of the Doppler shifts (including for example, whether EIS is able to resolve the red-and blueshifts due to the untwisting motion alone). Observations of similar events with improved spectral coverage should clarify what occurs in situations such as these.

Recent models of the minifilament jet-producing mechanism (Wyper et al. 2017, 2018) argue that "breakout reconnection" at a coronal magnetic null point between the pre-eruption field enveloping the minifilament and an ambient (nearly vertical) coronal field is essential for initiating minifilament eruptions that produce single-spire jets. While this may hold for the single-spire jets they modeled, initial breakout reconnection does not appear to be essential in the two-sided loop jet presented here; the minifilament flux-rope eruption undergoes clear acceleration before the overlying field (where "breakout" reconnection eventually occurred at the interface) abruptly stops its motion (Figure 5). Thus the possibility remains that tether-cutting (e.g., Moore \& LaBonte 1980), or an ideal magnetohydrodynamic instability (e.g., Török \& Kliem 2005), prior to the breakout reconnection might be the primary initiation mechanism for this minifilament eruption.

In summary, our view for this two-sided loop jet is that (a) magnetic flux cancelation built the minifilament field and triggered it to erupt, (b) the erupting-minifilament field pushed up into the largely horizontally oriented pre-existing magnetic field, (c) and the two-sided loop jet spires developed due to reconnection between the erupting-minifilament field and the overlying field.

Additionally, the central brightening in our event corresponds to the JBP of single-spire jets and is a miniature flare that accompanied the erupting minifilament; it is analogous to large-scale flares accompanying typically sized filament eruptions, according to the standard flare model (e.g., Hirayama 1974; Shibata et al. 1995; Moore et al. 2001). Brightening of the horizontal jet loops would be due to a combination of heating of material at the reconnection location, to a density increase from evaporation from the near and far ends of the reconnected far-reaching loops, and to a density increase from leakage onto the horizontal segments of material in the erupting-minifilament field (including the minifilament material itself).

While there are now numerous examples of minifilament eruptions driving (single-spire) coronal jets (e.g., Shen et al. 2012a; Moore et al. 2013; Sterling et al. 2015; Hong et al. 2016; Panesar et al. 2016; Zhang et al. 2016; Liu et al. 2017) it is necessary to study more than this single example of a twosided loop jet before we might conclude that two-sided loop jets, in general, are also driven by minifilament eruptions, as other recent observation do not discuss erupting minifilaments in two-sided loop jet formation (Tian et al. 2017; Zheng et al. 2018). Therefore more studies are needed to determine the most common cause of two-sided loop jets. 
We thank an anonymous referee for comments that improved the paper. This work was supported by funding from the Heliophysics Division of NASA's Science Mission Directorate through the Heliophysics Guest Investigator (HGI) Program, and the Hinode Project. Data used in this study were taken during Hinode 2015-2016 "focused mode" observations. Hinode is a Japanese mission developed and launched by ISAS/JAXA, with NAOJ as domestic partner and NASA and STFC (UK) as international partners. It is operated by these agencies in cooperation with ESA and NSC (Norway).

\section{Appendix A \\ Online Animations}

This Appendix collects together figures corresponding to online animations for figures in the text: Figures 8-14, respectively, correspond to text Figures 1(a)-(b), 1(c), 1(d)-(e), 1(f), 1(h), 1(i), and 3. Figures 8-14 are, therefore, redundant with the figures earlier in the text, but they are included to facilitate access to large-frame versions of the animations. No information will be lost if Figures $8-14$ are omitted when the reader prints out the paper in PDF format.

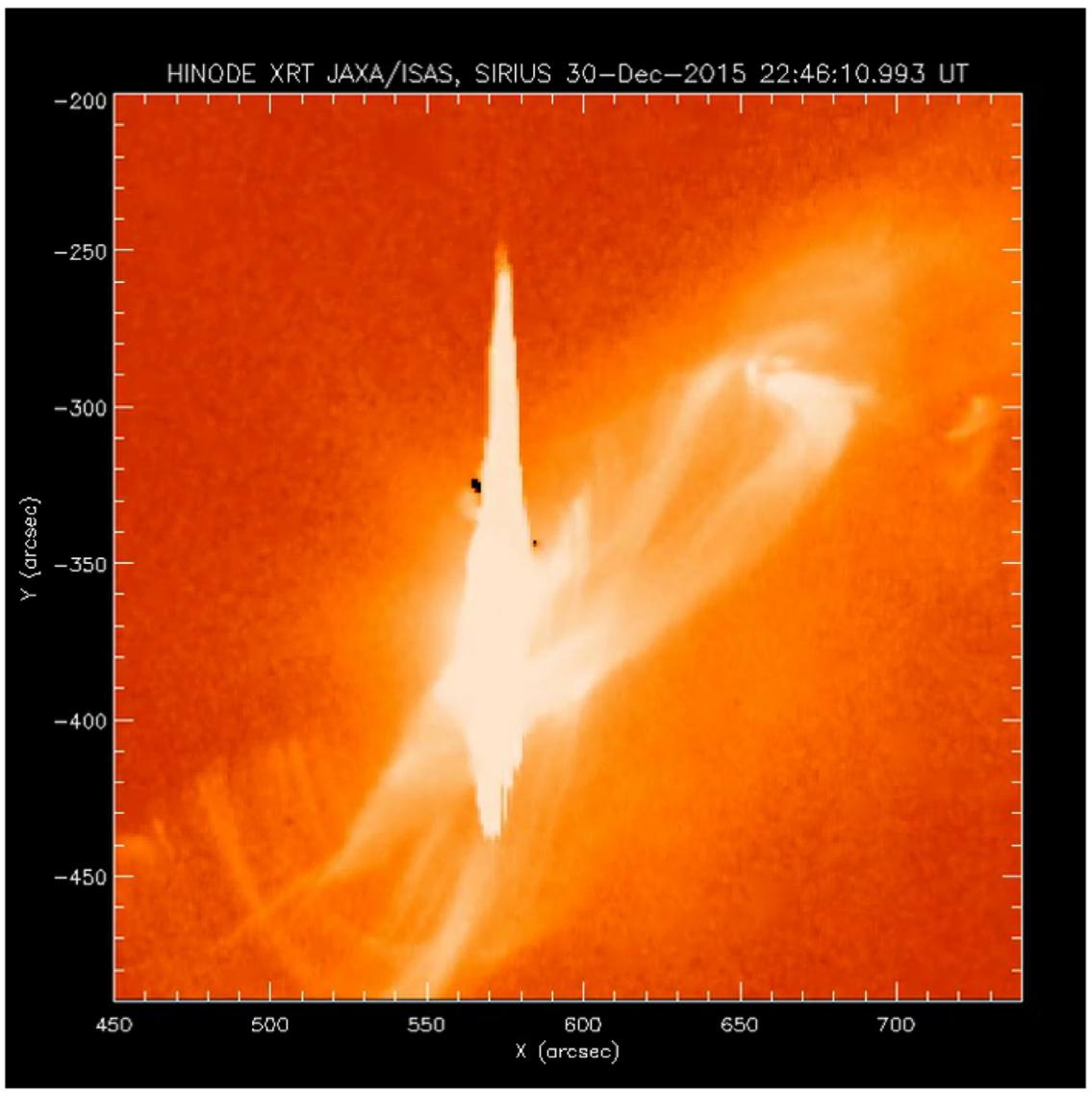

Figure 8. Online animated version of Figures 1(a)-(b): from Hinode/XRT, showing the two-sided loop jet from near the onset time to after peak intensity. The animation covers from 22:44 UT on 2015 December 30 to 01:14 UT on 2015 December 31.

(An animation of this figure is available.) 


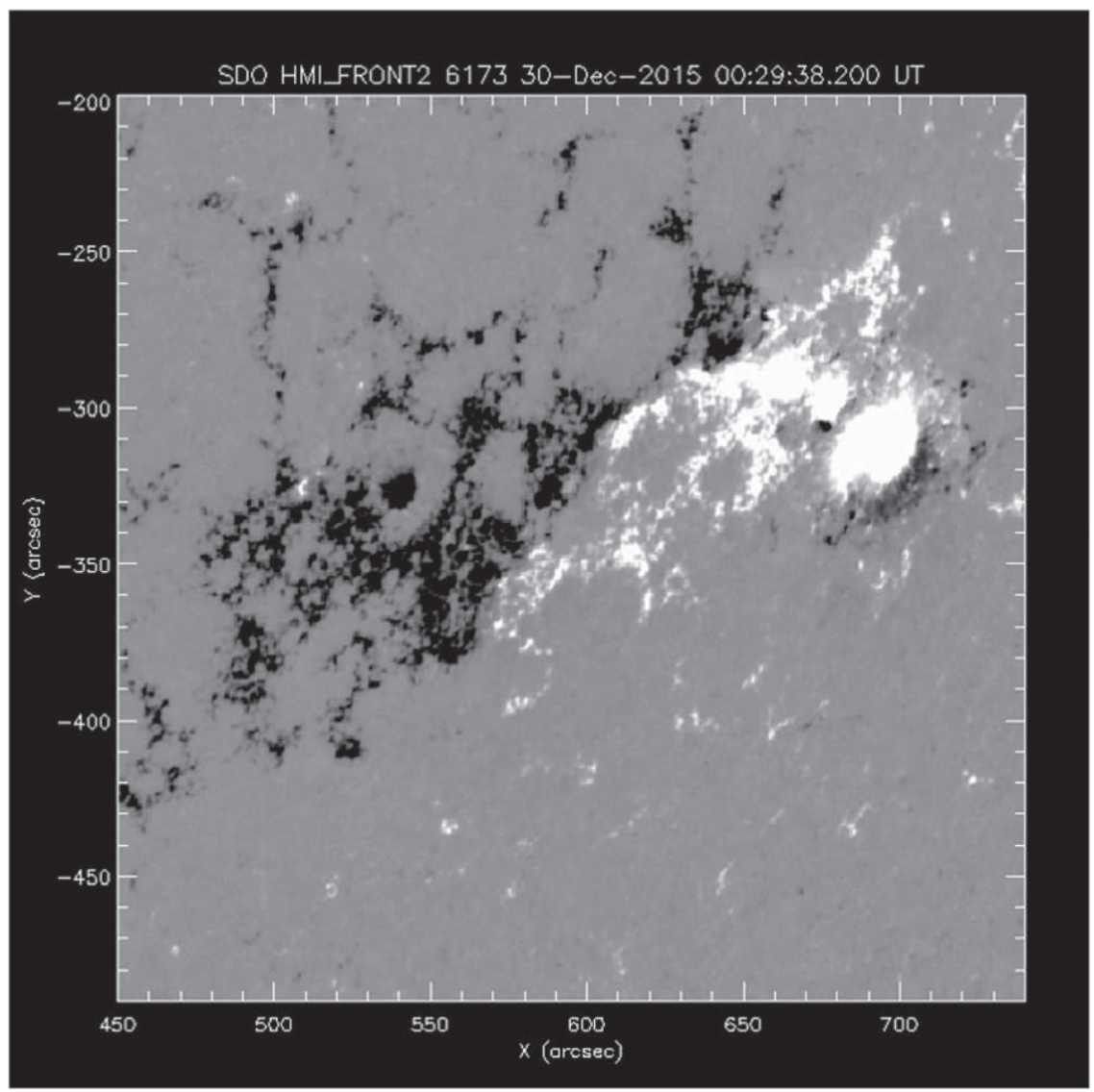

Figure 9. Online animated version of Figure 1(c): HMI magnetogram of the jet region, covering from 23:59 UT on 2015 December 29 to $11: 52$ UT on 2015 December 31

(An animation of this figure is available.) 


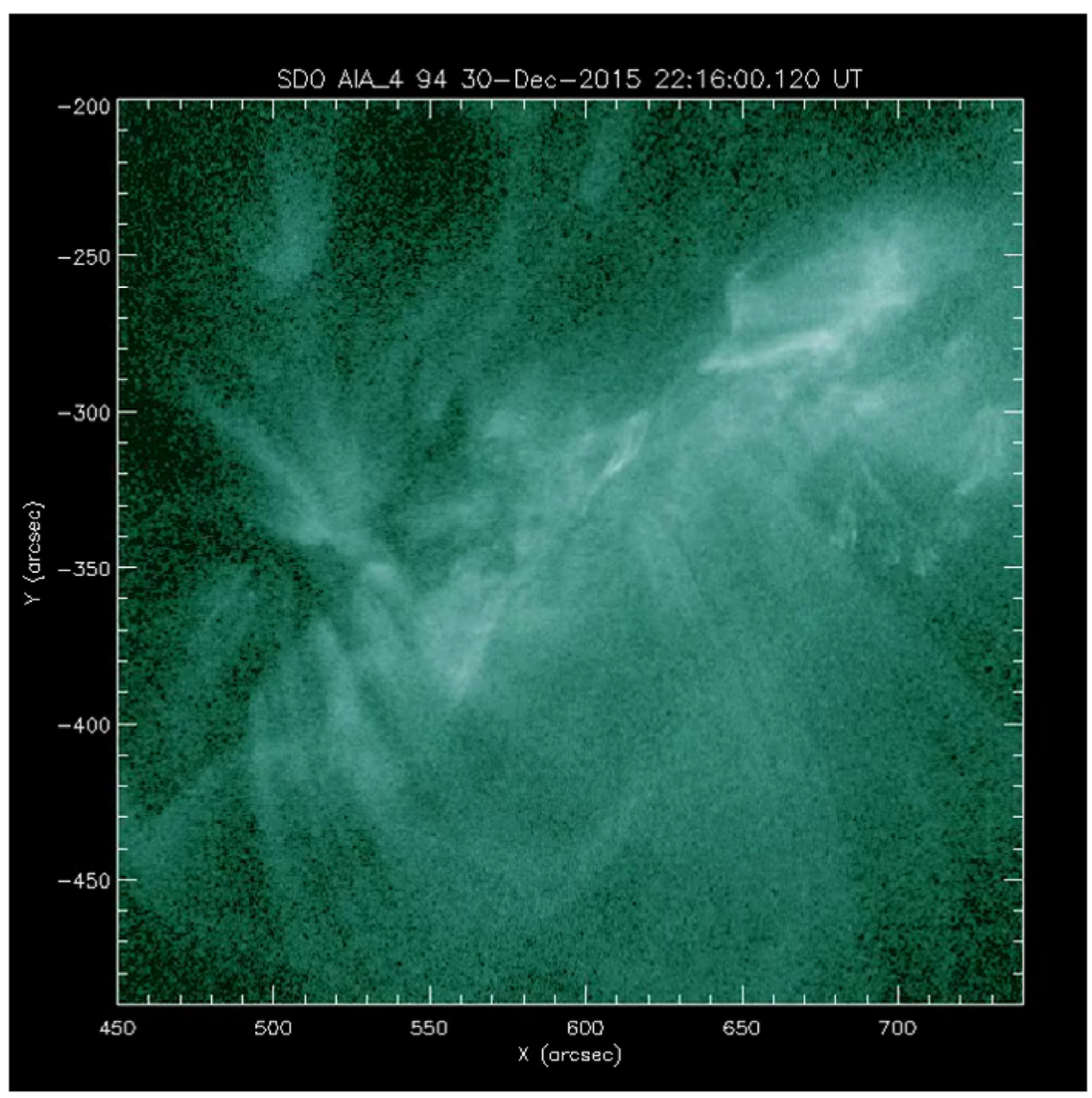

Figure 10. Online animated version of Figures 1(d)-(e): AIA $94 \AA$ Animation, covering from 22:15 to 23:15 UT on 2015 December 30. (An animation of this figure is available.) 


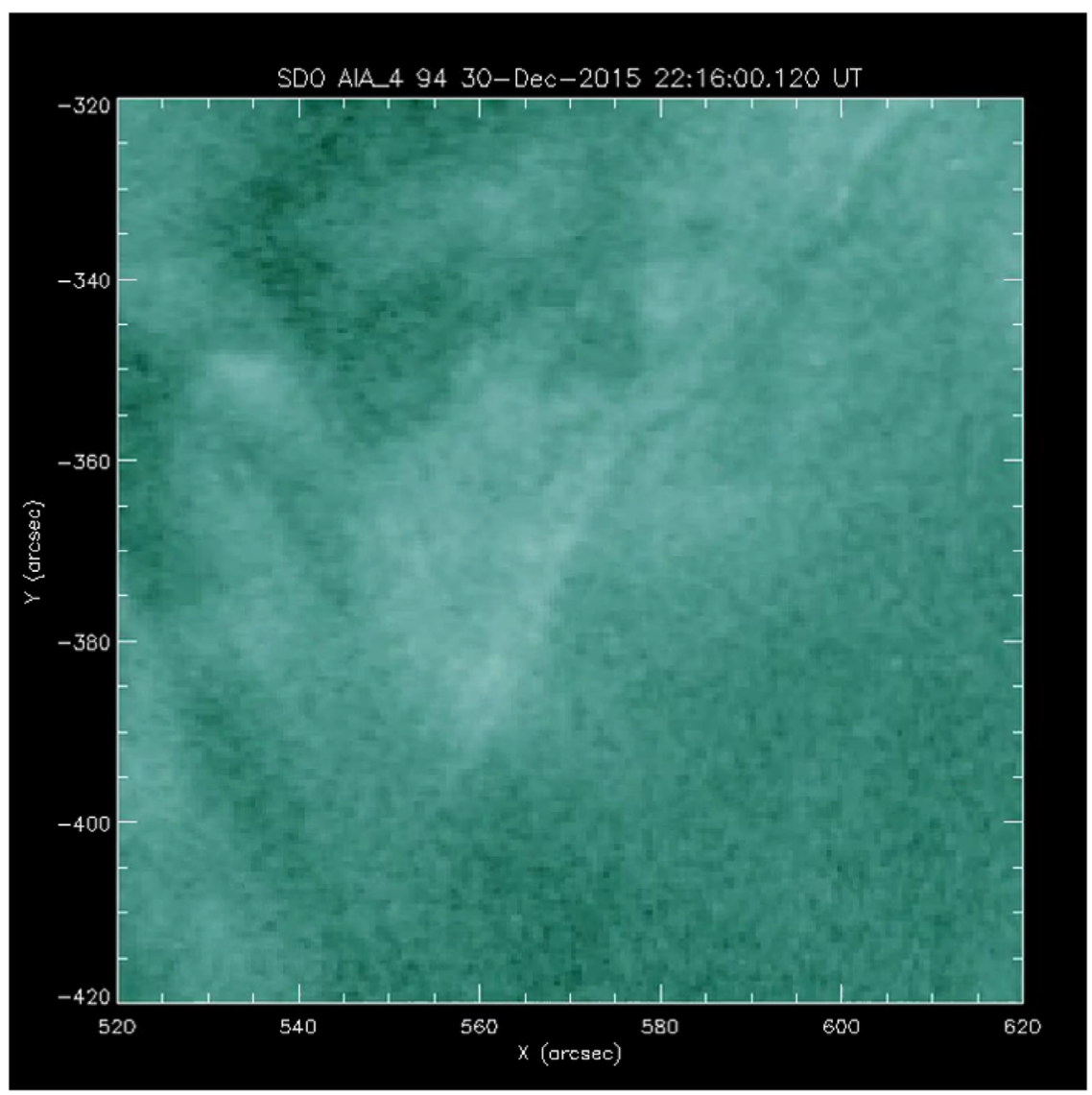

Figure 11. Online animated version of Figure 1(f): zoomed-in view of the AIA $94 \AA$ A images focused on the boxed area of Figure 1(d). The animation covers from 22:15 to 23:15 UT on 2015 December 30 .

(An animation of this figure is available.) 


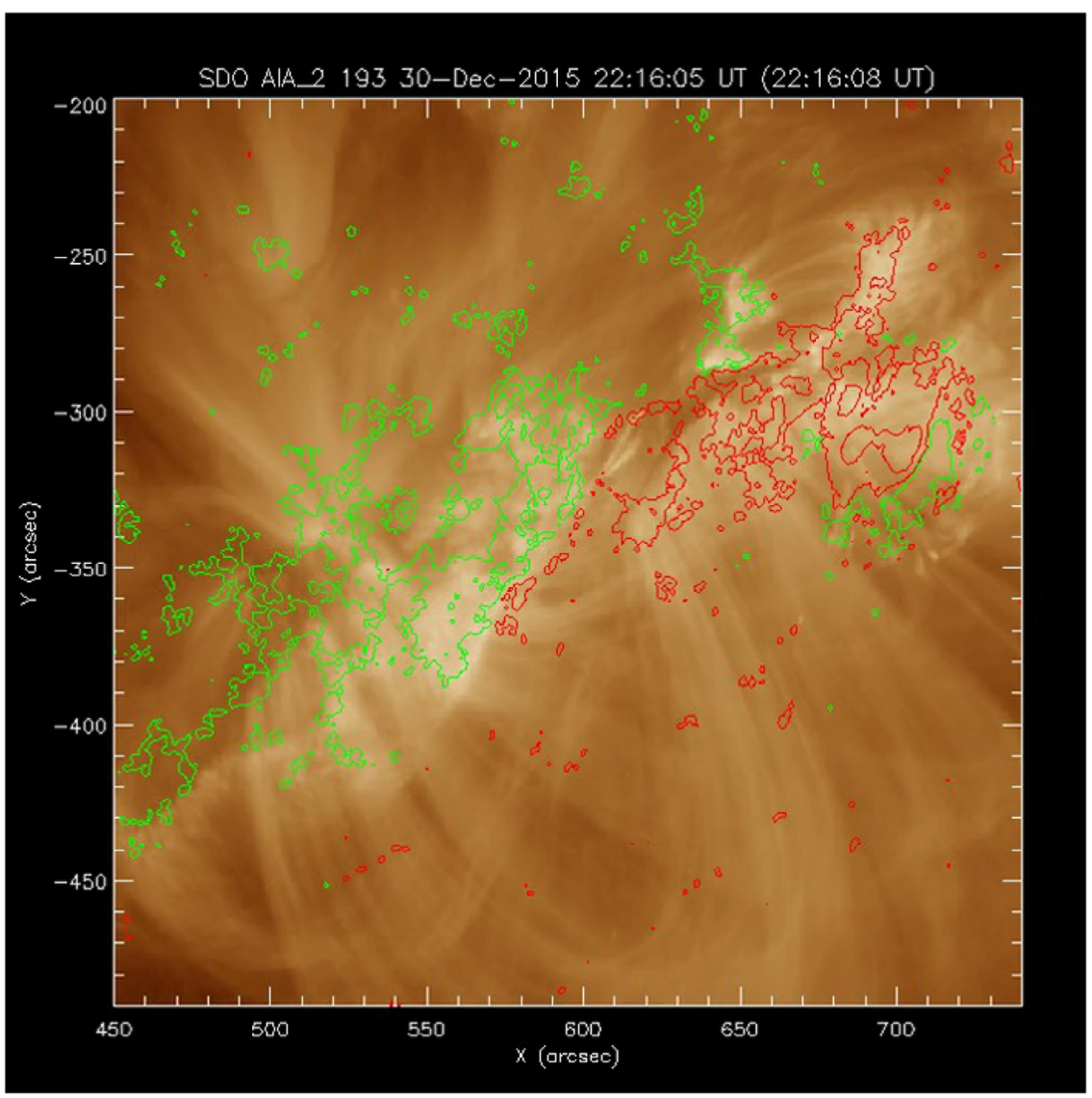

Figure 12. Online animated version of Figure 1(h): AIA $193 \AA$ A images, including the HMI magnetogram contours. The animation covers from 22:15 to 23:15 UT on 2015 December 30. The first times in the title are for the AIA image, and the times in parentheses in the title are for HMI.

(An animation of this figure is available.) 


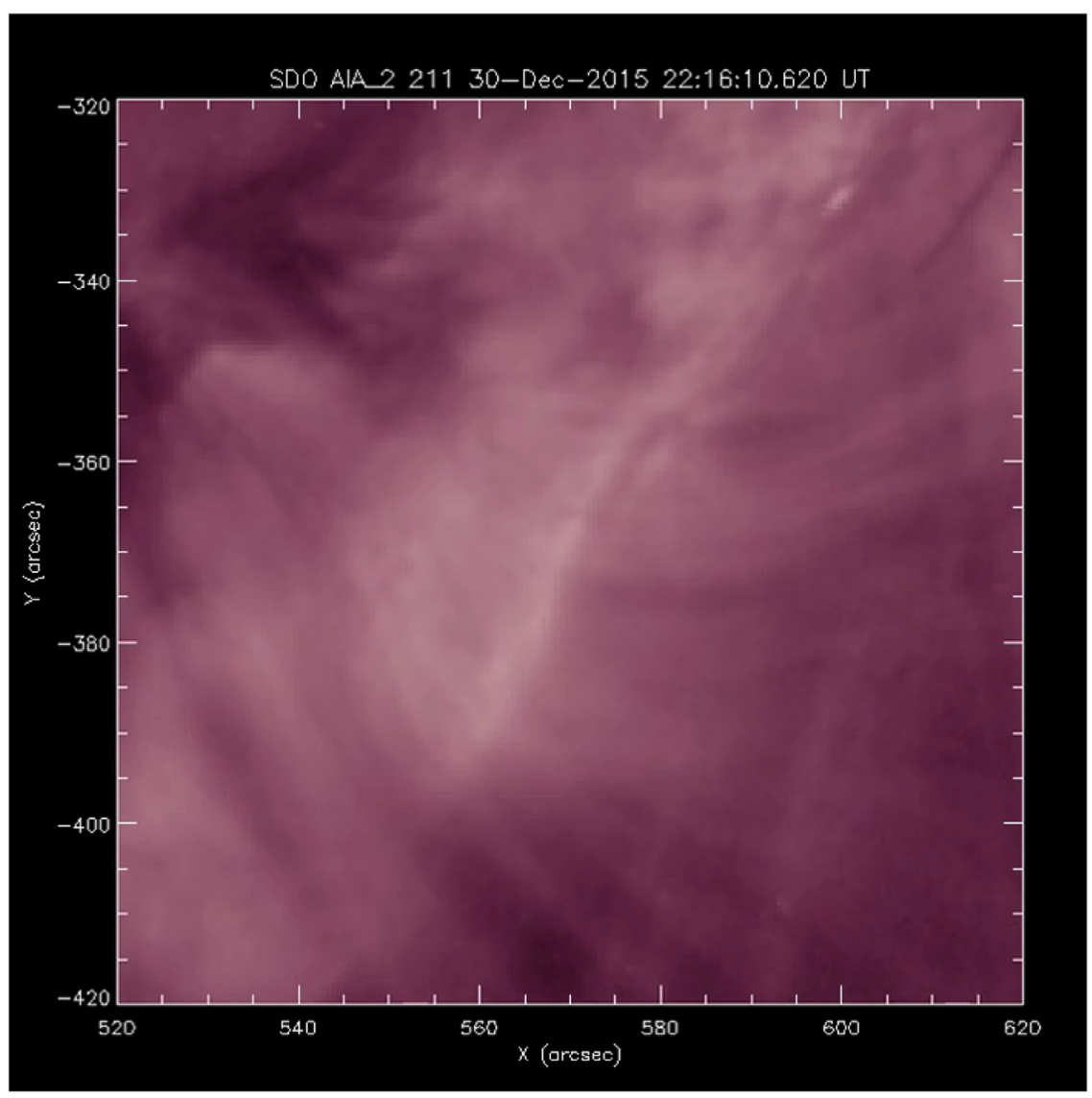

Figure 13. Online animated version of Figure 1(i): AIA $211 \AA$ A images. The animation covers from 22:15 to 23:15 UT on 2015 December 30. (An animation of this figure is available.) 


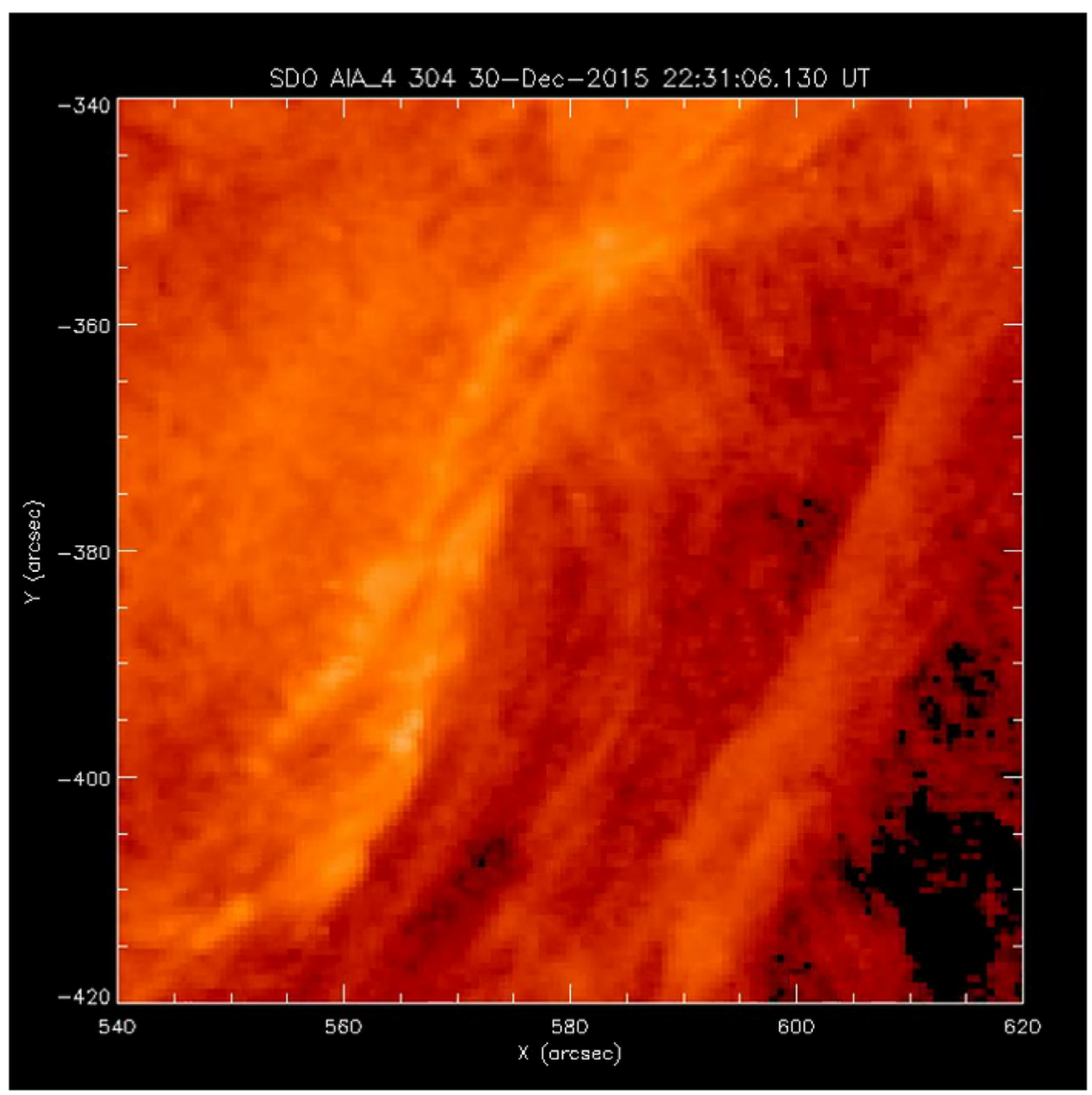

Figure 14. Online animated version of Figure 3: smaller FOV of the event of Figure 1, showing the minifilament eruption leading to the two-sided loop jet in AIA 304 $\AA$ images. The animation covers from 22:30 to 23:07 UT on 2015 December 30 .

(An animation of this figure is available.)

\section{ORCID iDs}

Alphonse C. Sterling (iD https://orcid.org/0000-00031281-897X

Louise K. Harra (1) https://orcid.org/0000-0001-9457-6200

\section{References}

Adams, M., Sterling, A. C., Moore, R. L., \& Gary, G. A. 2014, ApJ, 783, 11 Alexander, D., \& Fletcher, L. 1999, SoPh, 190, 167

Cirtain, J. W., Golub, L., Winebarger, A. R., et al. 2007, Sci, 318, 1580

Culhane, J. L., Harra, L. K., James, A. M., et al. 2007, SoPh, 243, 19

Del Zanna, G. 2008, A\&A, 481, L49

Doschek, G. A. 2012, ApJ, 754, 153

Doschek, G. A., Mariska, J. T., Warren, H. P., et al. 2007, PASJ, 59, S707

Golub, L., Deluca, E., Austin, G., et al. 2007, SoPh, 243, 63

Green, L. M., Török, T., Vršnak, B., Manchester, W., \& Veronig, A. 2016, SSRv, 214, 46

Harra, L. K., Hara, H., Doschek, G. A., et al. 2017, ApJ, 842, 58

Hirayama, T. 1974, SoPh, 34, 323

Hong, J., Jiang, Y., Yang, J., et al. 2016, ApJ, 830, 60

Huang, Z., Madjarska, M. S., Doyle, J. G., \& Lamb, D. A. 2012, A\&A, 548, A62

Hudson, H. S. 2000, ApJ, 531L, 75

Imada, S., Bamba, Y., \& Kusano, K. 2014, PASJ, 66, 17

Janse, A. M., \& Low, B. C. 2007, A\&A, 472, 957

Jiang, Y., Bi, Y., Yang, J., et al. 2013, ApJ, 775, 132

Lemen, J. R., Title, A. M., Akin, D. J., et al. 2012, SoPh, 275, 17

Li, X., Yang, S., Chen, H., \& Zhang, J. 2015, ApJ, 814, 13L

Liu, J., Wang, Y., Shen, C., et al. 2017, ApJ, 813, 115

McCauley, P. I., Su, Y. N., Schanche, N., et al. 2015, SoPh, 290, 1703

McKenzie, D. E. 2000, SoPh, 195, 381

Moore, R. L., Hurford, G. J., Jones, H. P., \& Kane, S. R. 1984, ApJ, 276, 379
Moore, R. L., \& LaBonte, B. 1980, in IAU Symp. 91, Solar and Interplanetary Dynamics, ed. M. Dryer \& E. Tandberg-Hanssen (Dordrecht: Reidel), 207 Moore, R. L., Sterling, A. C., Falconer, D. A., \& Robe, D. 2013, ApJ, 769, 134 Moore, R. L., Sterling, A. C., Hudson, H. S., \& Lemen, J. R. 2001, ApJ, 552,833

Nisticò, G., Bothmer, V., Patsourakos, S., \& Zimbardo, G. 2009, SoPh, 259, 87 Panesar, N. K., Innes, D. E., Tiwari, S. K., \& Low, B. C. 2013, A\&A, 549, 105 Panesar, N. K., Sterling, A. C., \& Moore, R. L. 2017, ApJ, 844, 131

Panesar, N. K., Sterling, A. C., \& Moore, R. L. 2018, ApJ, 853, 189 Panesar, N. K., Sterling, A. C., Moore, R. L., \& Chakrapani, P. 2016, ApJL, 832, L7

Parenti, S. 2014, LRSP, 11, 1

Raouafi, N. E., Patsourakos, S., Pariat, E., et al. 2016, SSRv, 201,

Savage, S. L., \& McKenzie, D. E. 2011, ApJ, 730, 98

Savage, S. L., McKenzie, D. E., \& Reeves, K. K. 2012, ApJL, 747L, 40

Savcheva, A., Cirtain, J. W., DeLuca, E. E., et al. 2007, PASJ, 59S, 771S

Scherrer, P. H., Schou, J., Bush, R. I., et al. 2012, SoPh, 275, 207

Shen, Y., Liu, Y. E., Su, J., \& Deng, Y. 2012a, ApJ, 745, 164

Shen, Y., Liu, Y. E., Su, J., \& Deng, Y. 2012b, ApJ, 750, 12

Shibata, K., Ishido, Y., Acton, L. W., et al. 1992, PASJ, 44L, 173

Shibata, K., Masuda, S., Shimojo, M., et al. 1995, ApJ, 451L, 83

Shibata, K., Nitta, N., Matsumoto, R., et al. 1994, in Proc. Int. Symp. Yohkoh Scientific Results, X-Ray Solar Physics from Yohkoh, ed. Y. Uchida et al. (Tokyo: Univ. Academy Press), 29

Shimojo, M., Hashimoto, S., Shibata, K., et al. 1996, PASJ, 48, 123

Sterling, A. C., Harra, L. K., \& Moore, R. L. 2007, ApJ, 669, 1359

Sterling, A. C., \& Moore, R. L. 2005, ApJ, 630, 1148

Sterling, A. C., Moore, R. L., Falconer, D. A., et al. 2016, ApJ, 821, 100

Sterling, A. C., Moore, R. L., Falconer, D. A., \& Adams, M. 2015, Natur, 523,437

Sterling, A. C., Moore, R. L., Falconer, D. A., \& Knox, J. M. 2014, ApJ, 788L, 20

Sterling, A. C., Moore, R. L., Falconer, D. A., Panesar, N. K., \& Martinez, F. 2017, ApJ, 844, 28 
Sterling, A. C., Moore, R. L., \& Panesar, N. K. 2018, ApJ, 864, 68

Tian, Z., Liu, Y., Shen, Y., et al. 2017, ApJ, 845, 94

Török, T., \& Kliem, B. 2005, ApJL, 630, L97

Wang, H., Ewell, M. W., Jr., Zirin, H., \& Ai, G. 1994, ApJ, 424, 436

Warren, H. P., O’Brien, C. M., \& Sheeley, N. R., Jr. 2011, ApJ, 742, 92

Wheatland, M. S., Melrose, D. B., \& Mastrano, A. 2018, ApJ, 864, 159

Williams, D. R., Harra, L. K., Brooks, D. H., Imada, S., \& Hansteen, V. H. 2011, PASJ, 61, 493
Wyper, P. F., Antiochos, S. K., \& DeVore, C. R. 2017, Natur, 544, 452

Wyper, P. F., DeVore, C. R., \& Antiochos, S. K. 2018, ApJ, 852, 98

Yokoyama, T., \& Shibata, K. 1995, Natur, 375, 42

Yokoyama, T., \& Shibata, K. 1996, PASJ, 48, 353

Young, P. R., \& Muglach, K. 2014, SoPh, 289, 3313

Zhang, Q. M., Li, D., Ning, Z. J., et al. 2016, ApJ, 827, 27

Zheng, R., Chen, Y., Huang, Z., et al. 2018, ApJ, 861, 108

Zwaan, C. 1987, ARA\&A, 25, 83 